\title{
General Psychiatry Mental health in the post-COVID-19 era: challenges and the way forward
}

To cite: Vadivel R, Shoib S, El Halabi S, et al. Mental health in the post-COVID-19 era: challenges and the way forward. General Psychiatry 2021;34:e100424. doi:10.1136/ gpsych-2020-100424

Received 10 0ctober 2020 Revised 23 November 2020 Accepted 23 December 2020

\section{Check for updates}

(c) Author(s) (or their employer(s)) 2021. Re-use permitted under CC BY-NC. No commercial re-use. See rights and permissions. Published by BMJ.

For numbered affiliations see end of article.

Correspondence to Dr Ramdas Ransing; ramdas_ransing123@yahoo. co.in

\section{INTRODUCTION}

The COVID-19 pandemic has posed a serious threat to global mental health. Multiple lines of evidence suggest that there is a varying yet considerable increase in mental health issues among the general population and vulnerable groups. ${ }^{1}{ }^{2}$ The aftermath is obscure and speculative from a social, economic, individual and public mental health perspective. Recently published studies support the existence of an emotional epidemic curve, describing a high probability of an increase in the burden of mental health issues in the postpandemic era. ${ }^{3} 4$ Furthermore, previous major public health emergencies showed that more than half of the population developed mental health problems and required mental health intervention. ${ }^{45}$ There is, therefore, an urgent need to reorganise existing mental health services to address the current unmet needs for mental health and to prepare for future challenges in the postpandemic era in terms of prevention and management.

\section{THE BURDEN OF MENTAL HEALTH ISSUES IN THE POST-COVID-19 PANDEMIC ERA}

The current evidence and published literature related to previous epidemics suggest that mental health issues may arise after the peak of the pandemic, with increased prevalence among the vulnerable population and people with risk factors (box 1). ${ }^{4}$ The surge in mental health issues may remain untreated or undiagnosed due to interrupted mental health services and other challenges for mental health services in the post-COVID-19 pandemic era.
CHALLENGES FOR MENTAL HEALTH SERVICES IN POST-COVID-19 PANDEMIC ERA

The paucity of human resources, infrastructure and burn-out of mental health professionals (MHPs)

In many countries, MHPs have been redeployed for the provision of medical services in COVID-19 care centres. ${ }^{6}$ MHPs and physicians working in COVID-19 services are experiencing an increased level of mental health issues owing to work stress and the death of patients and loved ones. ${ }^{78}$ If the mental health of MHPs remains unaddressed, then these professionals may not be able to provide efficient mental health services in the postpandemic era. In low and middle-income countries (LMICs) where MHPs are scarce, this could further widen the treatment gap for mental disorders. ${ }^{9} 10$

\section{Assessing mental health issues}

In the postpandemic era, it may be difficult to identify mental disorders aetiologically related to COVID-19 (eg, anxiety due to cytokine storm) owing to a lack of specific diagnostic or screening tools. ${ }^{11}$ The Diagnostic and Statistical Manual of Mental Disorders, Fifth Edition or International Classification of Diseases 10th Revision/Eleventh Revisionbased diagnostic interviews may under-report or over-report the underlying conditions.

The impact of misinformation ('the infodemic') In some countries, particularly LMICs, waves of misinformation about COVID-19 are going to persist owing to multiple reasons (eg, religious and/or political beliefs). Surprisingly, most countries are not well prepared for managing this infodemic. ${ }^{3}$ The inability to access accurate information will strain the individual's mental health and may lead to an increase in polarisation and the occurrence of hate crimes. ${ }^{12}$ 
Box 1 Mental health issues, vulnerable population and risk factors

1. Mental health issues: including grief reactions, substance use disorders, anxiety, sleep disorders, depression, suicides, post-traumatic stress disorders, panic disorders. ${ }^{4,25,26}$

- New-onset mental health issues: due to COVID-19-related stress, fear and loneliness; enduring neuropsychiatric symptoms or disorders (eg, acute ischaemic stroke, headache, dizziness, ataxia, delirium and seizures) of COVID-19 infection due to cytokine storms. $^{27}$

- Relapse of pre-existing mental illness: due to reduced access to therapeutic resources, disruption of therapies, service provision and social support. ${ }^{410}$

- Suicides: due to neuropsychiatric manifestations and the socioeconomic impact of COVID-19. ${ }^{4}$

- Other issues: COVID-19-related stigma, discrimination and hate crimes. $^{21,28}$

2. Vulnerable population: children and adolescents; elderly; unemployed and homeless persons; COVID-19 survivors; healthcare workers (HCWs); those with pre-existing psychiatric disorders; grass-roots workers; pregnant women; people with disabilities and chronic diseases; migrants; refugees; lesbian, gay, bisexual, transgender and queer (LGBTQ) community; racial and ethnic minorities. $^{7,21,29}$

3. Risk factors: the death of either parent, caregivers or loved ones, misinformation, loss of peer support because of closure of school or workplace, academic loss, medical comorbidities, uncertainties, stigma, prolonged isolation, social rejection, work stress, burnout, being in direct contact with active cases and facing economic burdens. $^{4,25}$

\section{Access to mental healthcare services}

Lack of preparedness, overburdened mental health services, increased prevalence of mental health issues and interrupted mental health services will limit access to mental healthcare facilities in the postpandemic era, particularly in LMICs. Many psychiatric facilities and outpatient departments are currently converted to manage COVID-19. ${ }^{13}$ Therefore, people with mental illness may not seek help from these services owing to a fear of infection. Many pharmaceutical industries have changed their focus to the preparation of COVID-19related drugs, vaccines and preventive kits (sanitisers), which may hamper the production of psychopharmacological drugs. Perceived job insecurity, financial problems and unemployment contribute to significant risks for psychiatric disorders and pose an important barrier in accessing mental healthcare.

\section{Psychotherapy}

The complex and ever-changing dynamics of the COVID-19 pandemic will be a challenge for psychotherapeutic services owing to a lack of physical and social connection. ${ }^{15}$ In LMICs, the dearth of telepsychiatric services will limit accessibility to psychotherapy. ${ }^{10}$

\section{Support systems}

Adults, children, adolescents and families are affected by the loss of the structured support found in schools, childcare facilities or physical workplaces. ${ }^{4}$ Rebuilding this extrafamilial system will be challenging.

\section{Public health paradox and injustice}

A substantial body of evidence suggests that people with the highest level of mental health needs often have the least access to services. ${ }^{16}$ In a post-COVID-19 pandemic era, it will get exaggerated owing to economic recession, strain on resources and unemployment.

\section{RECOMMENDATIONS FOR POSTPANDEMIC MENTAL HEALTH SERVICE PREPAREDNESS}

Because of the limited scientific understanding of the COVID-19 pandemic and mental health thus far, postpandemic preparedness is difficult. The pandemic is an unpredictable, irregular occurrence and its impact could be difficult to measure and explore. Considering this, we recommend using the components of the mental health preparedness and action framework (MHPAF) for postpandemic preparedness. MHPAF consists of five interlinked components, including preparation and coordination, monitoring and assessment, sustainability of mental healthcare services, infodemic management and communications. ${ }^{3}$ This framework has been used to evaluate pandemic preparedness in some countries like Kenya and the USA. ${ }^{17}$ However, postpandemic mental health preparedness could be more challenging in countries that are inadequately prepared for pandemics. In addition to preparing for the components of MHPAF, we suggest a few additional interventions for effective and efficient management of postpandemic psychiatric services.

\section{Mental healthcare delivery}

COVID-19 has affected mental healthcare delivery because of the redeployment of MHPs. We need to reconsider a few practical approaches or models of care for effective delivery in the postpandemic era.

\section{Telepsychiatry}

Telepsychiatry needs to be developed through a government-supported service platform centred on community health service centres to enable easier access to psychiatric care, especially among vulnerable populations (eg, the elderly). However, the digital divide, access to marginalised populations and poverty are major barriers to telepsychiatry services in LMICs. This could affect the feasibility and acceptability of telepsychiatry in many countries. Considering this challenge, it is imperative that healthcare workers reach out to patients and aim at equitable access of telepsychiatric facilities.

\section{Infodemic management}

More robust regulation of social media companies by non-partisan, non-corporate, global regulators is needed to clamp down on the spreading of fake news, anti-vaccine movement and polarising content. All countries should take stringent steps towards infodemic management 
by the formulation of guidelines for responsible media reporting. Additionally, infoveillance (information monitoring), building eHealth literacy and capacity, knowledge refinement and accurate and timely knowledge translation should be encouraged. ${ }^{18}$

\section{Integrative care}

National public health policies should be designed to provide integrated care for mental health in different settings such as hospitals, primary care services, communities, schools, universities, colleges and workplaces. ${ }^{19}$ Formalising liaison between these settings with mental health services would help to promptly identify and holistically address emerging mental health needs. Developing support groups, screening of at-risk groups, peer counselling services, establishing dedicated crisis helplines, preparation for long-term plans and expanding support services can facilitate early access to mental health needs.

\section{Community mental health services}

Community mental health services should be well prepared to screen, identify people at risk, provide psychological first aid and facilitate onward referral services. ${ }^{20}$ Primary healthcare workers and organisational gatekeepers (eg, pharmacists, geriatric caregivers and school teachers) should be trained to identify individuals at risk and direct them to proper evaluation and treatment.

\section{Human resources, education and training}

Current redeployment of, and potential burn-out among, MHPs in the COVID-19 setting is affecting preparations for the delivery of mental health services for the postpandemic era. Policymakers and stakeholders should consider this as a priority. In many countries (like India), grass-roots medical staff (Accredited Social Health Activist-ASHA, teacher) are playing an important role in prescreening and triage, door-to-door visits, follow-up and on-site screening of COVID-19. Therefore, grass-roots workers should be trained in identifying and managing pandemic-associated psychiatric and psychosocial issues.

\section{Formulate guidelines and protocols}

Many people have been exposed to similar health risks, isolation, grief and economic uncertainty, individually and with their families. Therefore, certain common themes should be used to formulate guidelines to improve access to care.

\section{Assessment and intervention}

The use of a toolkit or stepped care or matched care model through primary care physicians can improve the coverage of mental health services in the postpandemic era by allowing them to manage common mental disorders of mild severity. ${ }^{20}$

\section{Suicide prevention}

In anticipation of an increase in suicide rates, efforts should be made to reduce access to means (eg, more stringent gun control) and for better resourcing with suicide prevention agencies along with global decriminalisation of suicide/attempt(s). In addition, early screening for mental illness and treatment should be encouraged.

\section{Research}

Prospective cohort studies should be carried out to identify risk factors and exposure levels, track outcomes and compare outcomes among subgroups. These studies are important to monitor the effect of various interventions and strategies.

\section{Stigma and discrimination}

Interventions are needed to reduce stigmatisation and discrimination towards minority or vulnerable groups and to inform policy changes. ${ }^{21}{ }^{22}$ General and specific interventions should be directed towards identification of drivers (eg, misinformation), facilitators (eg, lack of regulations) and intersecting factors (eg, occupation such as healthcare workers) towards reducing stigma and discrimination. ${ }^{21}$

\section{Networks and services}

A multinational network of MHPs in collaboration with World Health Organisation (WHO) should be set up to enable the sharing of research and clinical practice paradigms in the post-COVID-19 era. This network should focus on building resilience both in the community and on an individual level.

\section{Approach for addressing postpandemic mental health and services}

Addressing emergent challenges with appropriate interventions could be challenging in many countries particularly in low-resource settings. Therefore, efforts should be taken for the prevention of mental health issues on a large scale and organisation of services for early identification of mental health issues. These approaches to mental healthcare prevention and treatment after the COVID-19 crisis can be classified as universal, selective or indicated. $^{2324}$

\section{Universal approach}

This is a population-wide intervention that will help reduce the overall burden of mental health issues (stress, anxiety and fear) through prevention; therefore, it is imperative to have a universal approach for each country (box 2).

\section{Selective approach}

It should be used for an individual having the risk factors for developing mental health issues. For example, a vulnerable population and individuals with risk factors mentioned above. A screening toolkit or guidelines should be developed to identify these groups of people. ${ }^{20}$

\section{Indicated approach}

It should be designed for individuals having signs and symptoms of the mental issues as mentioned above. This approach ought to be guided by well-defined guidelines 
Box 2 Focus of universal approach in the postpandemic era

Promoting mental health wellness and reducing distress through adequate sleep, healthy diet and exercise, mindfulness-based programmes (eg, yoga) and awareness about mental health issues. ${ }^{30,31}$

- Using traditional and social media for mental health awareness campaigns and to encourage individuals to seek help with responsible, transparent and timely media reporting. ${ }^{3}$

- Establishing community support for those at risk and encouraging to stay connected and maintain relationships.

- Establishing primary screening services for common mental health issues such as anxiety, depression and suicidal thoughts.

- Establishing the national suicide prevention helplines or other helplines. ${ }^{32}$

- Integrating basic mental health services into primary care for early identification of COVID-19-related mental health issues.

- Developing self-help resources and promoting healthy coping strategies.

- Ensuring financial support for people through governmental and non-governmental organisations (eg, loans and credit).

before the intervention. Some people with mental health issues might not seek help because of fear of COVID-19 infection, stigma and poor motivation. It is therefore important to identify these individuals through a network of hospitals and community health workers.

\section{Active outreach}

It can be helpful for people with a history of psychiatric disorders, COVID-19 survivors and older adults.

\section{CONCLUSION}

To conclude, there is an immediate need to identify the long-term mental health consequences of the COVID-19 pandemic. Clinicians, researchers and policymakers are expected to be prepared for these mental health issues in terms of assessment, interventions and the model of care in the postpandemic era.

\section{Author affiliations}

${ }^{1}$ Department of Mental Health and Addictions, Waikato District Health Board, Hamilton, New Zealand

${ }^{2}$ Department of Psychiatry, Jawahar Lal Nehru Memorial Hospital, Rainawari, Srinagar, Jammu and Kashmir, India

${ }^{3}$ Department of Narrative Medicine, Columbia University, New York, New York, USA

${ }^{4}$ Department of Psychiatry, American University of Beirut, Beirut, Lebanon

${ }^{5}$ El Demerdash Teaching Hospital, Ain Shams University, Cairo, Egypt

${ }^{6} \mathrm{Hospital}$ and University Clinical Service of Kosovo, Community-Based Mental Health Center and House for Integration, Prizren, Kosovo

${ }^{7}$ Medical Faculty Carl Gustav Carus, Technical University Dresden, Dresden, Germany

${ }^{8}$ Clinic for Psychiatry and Psychotherapy, Elblandklinikum Radebeul, Academic Hospital Technical University, Dresden, Germany

${ }^{9}$ Department of Psychiatry and Legal Medicine, Universidade Federal do Rio Grande do Sul, Porto Alegre, Rio Grande do Sul, Brazil

${ }^{10}$ National Institute of Mental Health and Neuro Sciences, Bangalore, Karnataka, India

${ }^{11}$ Department of Social and Community Health, School of Population Health, University of Auckland, Auckland, New Zealand
${ }^{12}$ Department of Psychiatry, BKL Walawalkar Rural Medical College, Ratnagiri, Maharashtra, India

${ }^{13}$ Department of Child and Adolescent Psychiatry, NYU Grossman School of Medicine, New York University, New York, New York, USA

${ }^{14}$ Department of Psychiatry, Faculty of Medicine, Prince of Songkla University, Songkhla, Thailand

${ }^{15}$ Department of Neuropsychiatry, University of Port Harcourt Teaching Hospital, Port Harcourt, Rivers State, Nigeria

${ }^{16}$ Early Psychosis Intervention Programme, Institute of Mental Health, Singapore

Twitter Sheikh Shoib @sk_shoaib22, Sarah El Halabi @selhalabi1, Samer El Hayek @samerelhayek, Lamiaà Essam @lamiaessam14, Ramdas Ransing @ ram_ransing, Victor Pereira-Sanchez @victorpsanchez and Frances Nkechi Adiukwu @franadiukwu

Acknowledgements The authors wish to thank the Early Career Psychiatrists Section of the World Psychiatric Association (WPA) for being a supportive network that allowed early career psychiatrists from different countries to work together on this initiative.

Contributors RV, GKK and RAR developed the concept of this article. SS, RAR, RV, GKK, SHB, SHK, LE, RK, ALST, SN, RDR, VPS, CJ and FNA contributed to writing the first draft of the manuscript. All the authors reviewed the final version of the manuscript before submission. RAR is responsible for the overall content of this paper.

Funding The authors have not declared a specific grant for this research from any funding agency in the public, commercial or not-for-profit sectors.

Competing interests None declared.

Patient consent for publication Not required.

Provenance and peer review Not commissioned; externally peer reviewed.

Open access This is an open access article distributed in accordance with the Creative Commons Attribution Non Commercial (CC BY-NC 4.0) license, which permits others to distribute, remix, adapt, build upon this work non-commercially, and license their derivative works on different terms, provided the original work is properly cited, appropriate credit is given, any changes made indicated, and the use is non-commercial. See: http://creativecommons.org/licenses/by-nc/4.0/.

ORCID iDs

Sheikh Shoib http://orcid.org/0000-0002-3739-706X

Ramdas Ransing http://orcid.org/0000-0002-5040-5570

Chonnakarn Jatchavala http://orcid.org/0000-0001-9765-2184

Frances Nkechi Adiukwu http://orcid.org/0000-0001-5543-1746

\section{REFERENCES}

1 Qiu J, Shen B, Zhao M, et al. A nationwide survey of psychological distress among Chinese people in the COVID-19 epidemic: implications and policy recommendations. Gen Psychiatr 2020;33:e100213.

2 Wu M, Xu W, Yao Y. Mental health status of students' parents during COVID-19 pandemic and its influence factors. General Psychiatry;33.

3 Ransing R, Adiukwu F, Pereira-Sanchez V, et al. Mental health interventions during the COVID-19 pandemic: a conceptual framework by early career psychiatrists. Asian J Psychiatr 2020;51:102085.

4 Ren F-F, Guo R-J, Department of Neurology, Dongfang Hospital, Beijing University of Chinese Medicine, Beijing, China. Public mental health in POST-COVID-19 era. Psychiatr Danub 2020;32:251-5.

5 Taylor S, Asmundson GJG. Life in a post-pandemic world: what to expect of anxiety-related conditions and their treatment. J Anxiety Disord 2020;72:102231.

6 Pereira-Sanchez V, Adiukwu F, El Hayek S, et al. COVID-19 effect on mental health: patients and workforce. Lancet Psychiatry 2020;7:e29-30

7 Spoorthy MS, Pratapa SK, Mahant S. Mental health problems faced by healthcare workers due to the COVID-19 pandemic-A review. Asian J Psychiatr 2020;51:102119.

8 Greenberg N. Mental health of health-care workers in the COVID-19 era. Nat Rev Nephrol 2020;16:425-6.

9 Ransing RS, Agrawal G, Bagul K, et al. Inequity in distribution of psychiatry trainee seats and Institutes across Indian states: a critical analysis. J Neurosci Rural Pract 2020;11:299-308. 
10 Ramalho R, Adiukwu F, Gashi Bytyçi D, Bytyçi DG, et al. Telepsychiatry during the COVID-19 pandemic: development of a protocol for telemental health care. Front Psychiatry 2020;11:552450.

11 Ransing R, Ramalho R, Orsolini L, et al. Can COVID-19 related mental health issues be measured? Brain Behav Immun 2020;88:32-4.

12 Santé $\mathrm{WHO}=\mathrm{O}$ mondiale de la. Infodemic management: a key component of the COVID-19 global response - Parer aux infodémies: un élément essentiel de la riposte mondiale la COVID-19. Weekly Epidemiological Record = Relevé épidémiologique hebdomadaire 2020;95:145-8.

13 Bojdani E, Rajagopalan A, Chen A, et al. COVID-19 pandemic: impact on psychiatric care in the United States. Psychiatry Res 2020;289:113069.

14 Muruganandam P, Neelamegam S, Menon V, et al. COVID-19 and severe mental illness: impact on patients and its relation with their awareness about COVID-19. Psychiatry Res 2020;291:113265.

15 Plakun EM. Psychotherapy in the Post-COVID-19 era. J Psychiatr Pract 2020;26:309-12.

16 Vijayaraghavan M, Tochterman A, Hsu E, et al. Health, access to health care, and health care use among homeless women with a history of intimate partner violence. $J$ Community Health 2012;37:1032-9.

17 Jaguga F, Kwobah E. Mental health response to the COVID-19 pandemic in Kenya: a review. Int J Ment Health Syst 2020;14:68.

18 Eysenbach G. How to fight an Infodemic: the four pillars of Infodemic management. J Med Internet Res 2020;22:e21820.

19 Peng D, Wang Z, Xu Y. Challenges and opportunities in mental health services during the COVID-19 pandemic. Gen Psychiatr 2020;33:e100275.

20 Adiukwu F, Orsolini L, Gashi Bytyçi D, et al. COVID-19 mental health care toolkit: an international collaborative effort by early career psychiatrists section. Gen Psychiatr 2020;33:e100270.
21 Ransing R, Ramalho R, de Filippis R, et al. Infectious disease outbreak related stigma and discrimination during the COVID-19 pandemic: drivers, facilitators, manifestations, and outcomes across the world. Brain Behav Immun 2020;89:555-8.

22 Prasad SK, Karahda A, Singh P, et al. Role of mental health professionals in dealing with the stigma attached to COVID-19. Gen Psychiatr 2020;33:e100298.

23 Sher $\mathrm{L}$. The impact of the COVID-19 pandemic on suicide rates. QJM: An International Journal of Medicine, 2020: hcaa202.

24 Sher L. Preventing suicide. QJM 2004;97:677-80.

25 Das N. Psychiatrist in post-COVID-19 era - Are we prepared? Asian J Psychiatr 2020;51:102082

26 Vindegaard N, Benros ME. COVID-19 pandemic and mental health consequences: Systematic review of the current evidence. Brain Behav Immun;89:531-42.

27 Raony Ícaro, de Figueiredo CS, Pandolfo P, et al. PsychoNeuroendocrine-Immune interactions in COVID-19: potential impacts on mental health. Front Immunol 2020;11:1170.

28 Varatharaj A, Thomas N, Ellul MA, et al. Neurological and neuropsychiatric complications of COVID-19 in 153 patients: a UKwide surveillance study. Lancet Psychiatry 2020;7:875-82.

29 Cabarkapa S, Nadjidai SE, Murgier J, et al. The psychological impact of COVID-19 and other viral epidemics on frontline healthcare workers and ways to address it: a rapid systematic review. Brain Behav Immun Health 2020;8:100144.

30 Ransing R, Pinto da Costa M, Adiukwu F, et al. Yoga for COVID-19 and natural disaster related mental health issues: challenges and perspectives. Asian J Psychiatr 2020;53:102386.

31 Nagendrappa S, Shoib S, Rehman S, et al. Recognizing the role of animal-assisted therapies in addressing mental health needs during the COVID-19 pandemic. Asian J Psychiatr 2020;53:102390.

32 Ransing R, Kar SK, Menon V. National helpline for mental health during COVID-19 pandemic in India: new opportunity and challenges ahead. Asian J Psychiatr 2020;54:102447.

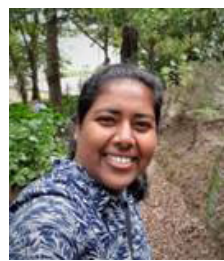

Dr. Ramyadarshni Vadivel obtained her MBBS degree from the Jawaharlal Institute of Postgraduate Medical Education and Research (JIPMER), Pondicherry, India, in 2008. Now, she is an advanced trainee in mental health and addictions of the Royal Australian and New Zealand College of Psychiatrists (RANZCP). She is also working as a senior registrar in the addictions services of Waikato District Health Board (DHB) in Hamilton, New Zealand. She has been working in Waikato DHB since December 2017 and training in RANZCP since June 2016. She is a member of the Early Career Psychiatrist (ECP) Section of the World Psychiatric Association (WPA) and Network of Early Career Professionals working in the area of Addiction Medicine (NECPAM). In addition, she is a recipient of the World Congress of Social Psychiatry Young Fellow (WCSPYF) (2013), Japan Young Psychiatrists Organisation (JYPO) Fellowship (2013), and WPA ECP fellowship (2018), among other academic awards. Her main research interests include neurostimulation in the treatment of psychiatric disorders, with a special interest in addictions, smartphones and the use of technology in psychiatry and psychiatry in special populations (migration and refugees). She is currently a member of the worldwide network of ECP researchers working on the impact of the COVID-19 pandemic on different areas of mental health and addictions. 